\title{
THE VIBRATION OF THE STRING WITH THE INTERSTITIAL MASSIVE POINT
}

\author{
Miroslav Pardy \\ Department of Physical Electronics \\ Masaryk University \\ Kotlářská 2, 61137 Brno, Czech Republic \\ e-mail:pamir@physics.muni.cz
}

May 20, 2021

\begin{abstract}
We will consider the string, the left end of which is fixed at the beginning of the coordinate system, the right end is fixed at point $l$ and mass $m$ is fixed between the ends of the string. We determine the vibration of such system. The proposed model can be also related in the modified form to the problem of the Mössbauer effect, or recoilless nuclear resonance fluorescence, which is the resonant and recoilfree emission and absorption of gamma radiation by atomic nuclei bound in a solid. (Mössbauer,1958).
\end{abstract}

\section{Introduction}

We will consider the string, the left end of which is fixed at the beginning of the coordinate system, the right end is fixed at point $l$ and mass $m$ is fixed interstitially between the ends of the string. The vibration motion of the string and the massive point with mass $m$ is the problem of the mathematical physics in case that the tension is linearly dependent on elongation.

The differential equation of motion of string elements can be derived by the well known way. We suppose that the string tension force acting on the element $d l$ of the string is given by the law (Tikhonov et al., 1977):

$$
T(x, t)=E S\left(\frac{\partial u}{\partial x}\right),
$$

where $E$ is the modulus of elasticity, $S$ is the cross section of the string. We easily derive that 


$$
T(x+d x)-T(x)=E S\left(\frac{\partial u}{\partial x}\right)(x+d x)-E S\left(\frac{\partial u}{\partial x}\right)(x) \approx E S u_{x x} d x
$$

The mass $d m$ of the element $d l$ is $\varrho S d x$, where $\varrho$ is the mass density of the string matter and the dynamical equilibrium gives

$$
\varrho S d x u_{t t}=E S u_{x x} d x .
$$

Or, after minimal modification we get

$$
\frac{1}{c^{2}} u_{t t}-u_{x x}=0 ; \quad c=\left(\frac{E}{\varrho}\right)^{1 / 2} .
$$

The last procedure was performed evidently in order to get the wave equation.

\section{The string motion with the interstitial massive point}

Now, let us consider the string with the point-like mass at coordinate $s$ in the interval $(0, l)$. Then, the left part string motion of the string be $u_{1}(x, t)$ and the right side of the string motion is $u_{2}(x, t)$. The corresponding equation of motion of both part of the string are as follows:

$$
\begin{aligned}
& \left(u_{1}\right)_{t t}=c^{2}\left(u_{1}\right)_{x x} ; \quad(0<x<s), \\
& \left(u_{2}\right)_{t t}=c^{2}\left(u_{2}\right)_{x x} ; \quad(s<x<l) .
\end{aligned}
$$

The boundary and interstitial conditions are with $S=1$ :

$$
\begin{gathered}
u_{1}(x=0)=0, \quad u_{2}(x=l)=0 . \\
u_{1}(x=s)=u_{2}(x=s) .
\end{gathered}
$$

The dynamical equation involving interstitial point is with $E=\varrho c^{2}$ :

$$
\varrho c^{2}\left(u_{1}\right)_{x}(s)-\varrho c^{2}\left(u_{2}\right)_{x}(s)=m\left(u_{1}\right)_{t t}(s)-m\left(u_{2}\right)_{t t}(s) .
$$

Let us look for the solution of the last equation in the form

$$
\begin{gathered}
u_{1}(x, t)=C_{1} \sin \frac{\omega x}{c} \sin \omega t \\
u_{2}(x, t)=C_{2} \sin \frac{\omega(l-x)}{c} \sin \omega t .
\end{gathered}
$$

We see that the suggested solution is in harmony with the boundary conditions:

$$
u_{1}(x=0)=0, \quad u_{2}(x=l)=0 .
$$


After insertion of $u_{1}(x, t), u_{2}(x, t)$ from (9-10) into eqs. (7-8), we get the system of equations

and

$$
C_{1} \sin \frac{\omega s}{c}=C_{2} \sin \frac{\omega(l-s)}{c}
$$

$$
\begin{aligned}
& C_{1} \varrho c \omega \cos \frac{\omega s}{c}-C_{2} \varrho c \omega \cos \frac{\omega(l-s)}{c}= \\
& C_{1} m \omega^{2} \sin \frac{\omega s}{c}-C_{2} m \omega^{2} \sin \frac{\omega(l-s)}{c} .
\end{aligned}
$$

In order to get the regular solution, the determinant of the system must be zero. Or,

$$
\left|\begin{array}{ll}
A & B \\
C & D
\end{array}\right|=0
$$

where

$$
\begin{gathered}
A=\sin \frac{\omega s}{c} \\
B=-\sin \frac{\omega(l-s)}{c} \\
C=\varrho c \omega \cos \frac{\omega s}{c}-m \omega^{2} \sin \frac{\omega s}{c} \\
D=-\varrho c \omega \cos \frac{\omega(l-s)}{c}+m \omega^{2} \sin \frac{\omega(l-s)}{c} .
\end{gathered}
$$

It follows from eq. (14)

$$
\left|\begin{array}{ll}
A & B \\
C & D
\end{array}\right|=A D-B C=0
$$

Or,

$$
\begin{gathered}
\sin \frac{\omega s}{c} \cos \frac{\omega(l-s)}{c}\left[-\varrho c \omega+m \omega^{2} \tan \frac{\omega(l-s)}{c}\right]+ \\
\sin \frac{\omega(l-s)}{c} \cos \frac{\omega s}{c}\left[\varrho c \omega-m \omega^{2} \tan \frac{\omega s}{c}\right]=0,
\end{gathered}
$$

So, we see, that the determination of the frequency $\omega$ involves the trancendent equation. The solution can be performed graphically, or by computer. Such problem is the integral part of the university mathematical methods (Arfken, 1967).

Nevertheless, it is evident that the trivial solution is for $\omega=0$ and for

$$
\omega s=\pi n c, \quad n=0,1,2, \ldots ; \quad \omega(l-s)=\pi k c, k=0,1,2, \ldots,
$$

which implies that the correspondence between $l$ and $k$ is only for

$$
\frac{l}{s}=\frac{(k+n)}{n} \text {. }
$$

The determination of the $\omega$ from the transcendent equations

$$
\left[-\varrho c \omega+m \omega^{2} \tan \frac{\omega(l-s)}{c}\right]=0, \quad\left[\varrho c \omega-m \omega^{2} \tan \frac{\omega s}{c}\right]=0
$$

is difficult and it can be solved by the appropriate mathematical methods (Arfken, 1967). 


\section{Discussion}

The articles was inspired by the author university elaborate (Pardy, 1965), in which the interaction of light with the crystal defect was calculated. At this elaborate the crystal was replaced by the Euler-Bernoulli linear chain (Landau, et al., 1965) with some defects.

The proposed model can be also related in the modified form to the problem of the Mössbauer effect, or recoilless nuclear resonance fluorescence, which is the resonant and recoil-free emission and absorption of gamma radiation by atomic nuclei bound in a solid. (Mössbauer,1958). In this effect, a narrow resonance for nuclear gamma emission and absorption results from the momentum of recoil transited to a surrounding crystal lattice and not to the emitting or absorbing nucleus alone. No gamma energy is lost. Emission and absorption occur at the same energy, resulting in strong, resonant absorption.

The generalization of our continual model can be performed in such a way that we replace one massive point $m$ by the massive points $m_{1}, m_{2}, m_{3}, \ldots \quad m_{k}$ at coordinates $s_{1}, s_{2}, s_{3}, \ldots \quad s_{k}$ and solve the adequate system of differential equations.

\section{References}

Landau, L. D. and Lifschitz, E. M. Mechanics (Nauka, Moscow, 1965).(in Russian).

Mössbauer, R. L. (1958).Kernresonanzfluoreszenz von Gammastrahlung in Ir191. Zeitschrift für Physik A (in German). 151 (2) 124-143

Pardy, M. (1965). The interaction of light with the crystal, Diploma work, (The Library of the University of J. E. Purkynje, Brno, Czech republic), (in Czech).

Tikhonov, A. N. and Samarskii, A. A. The Equations of Mathematical Physics (Nauka, Moscow, 1977). (in Russsian).

Arfken, G. Mathematical Methods for Physicists (Academic Press, New York and London, 1967). 